\title{
On steady poloidal and toroidal flows in tokamak plasmas
}

\author{
K. G. McClements ${ }^{1}$ and M. J. Hole ${ }^{2}$ \\ ${ }^{1}$ Culham Science Centre, EURATOM/CCFE Fusion Association, Abingdon, \\ Oxfordshire OX14 3DB, United Kingdom \\ ${ }^{2}$ Department of Theoretical Physics, Research School of Physical Science and Engineering, \\ Australian National University, Canberra ACT 0200, Australia
}

(Received 13 April 2010; accepted 6 July 2010; published online 23 August 2010)

\begin{abstract}
The effects of poloidal and toroidal flows on tokamak plasma equilibria are examined in the magnetohydrodynamic limit. "Transonic" poloidal flows of the order of the sound speed multiplied by the ratio of poloidal magnetic field to total field $B_{\theta} / B$ can cause the (normally elliptic) GradShafranov (GS) equation to become hyperbolic in part of the solution domain. It is pointed out that the range of poloidal flows for which the GS equation is hyperbolic increases with plasma beta and $B_{\theta} / B$, thereby complicating the problem of determining spherical tokamak plasma equilibria with transonic poloidal flows. It is demonstrated that the calculation of the hyperbolicity criterion can be easily modified when the assumption of isentropic flux surfaces is replaced with the more tokamak-relevant one of isothermal flux surfaces. On the basis of the latter assumption, a simple expression is obtained for the variation of density on a flux surface when poloidal and toroidal flows are simultaneously present. Combined with Thomson scattering measurements of density and temperature, this expression could be used to infer information on poloidal and toroidal flows on the high field side of a tokamak plasma, where direct measurements of flows are not generally possible. It is demonstrated that there are four possible solutions of the Bernoulli relation for the plasma density when the flux surfaces are assumed to be isothermal, corresponding to four distinct poloidal flow regimes. Finally, observations and first principles-based theoretical modeling of poloidal flows in tokamak plasmas are briefly reviewed and it is concluded that there is no clear evidence for the occurrence of supersonic poloidal flows. (C) 2010 American Institute of Physics.
\end{abstract}

[doi:10.1063/1.3469580]

\section{INTRODUCTION}

Poloidal and toroidal flows in tokamak plasmas are of great interest, primarily because they are known to affect both neoclassical ${ }^{1-3}$ and turbulent ${ }^{4-6}$ transport, and also magnetohydrodynamic (MHD) stability. ${ }^{7-9}$ A strong correlation has been observed between highly sheared poloidal flows and internal transport barriers in several tokamaks, notably in the Joint European Torus (JET) ${ }^{10}$ One of the challenges created by the presence of strong flows is that the traditional approach to modeling plasma equilibria, based on the assumption that inertial terms in the MHD force balance equation can be neglected, is no longer adequate. The GradShafranov (GS) equation, which describes flow-free axisymmetric equilibria, can be readily generalized to include both toroidal and poloidal flows when it is supplemented with an energy equation, which is required to provide closure. In many analyses of this problem, ${ }^{11-18}$ an isentropic energy equation has been employed, i.e., plasma entropy per unit mass has been assumed to be constant on a flux surface. While this assumption may be reasonable for some collisiondominated astrophysical plasmas, it is not generally appropriate for present-day tokamaks, in which the collisionality is sufficiently low that electron and ion temperatures are much more likely than specific entropy to be well-approximated by flux functions (although it has been argued that significant flux surface variations of ion temperature could occur in the presence of toroidal flows approaching the ion thermal speed $\left.^{19}\right)$. Guazzotto and co-workers ${ }^{16,18}$ discussed the flow equilibrium problem with both isothermal and isentropic closure conditions, but only carried out detailed analytical and numerical investigations in the isentropic case. Iacono and co-workers ${ }^{20}$ considered flow equilibria for various closure models, including one in which electron and ion temperatures parallel to the magnetic field (but not the perpendicular temperatures) were flux functions; for this closure scenario, it was shown that field-aligned flows produce a shift of the density profile toward the high field side of the plasma.

It has been recognized by several authors ${ }^{11,12,15,16,20,21}$ that whereas the GS equation in the absence of flows is elliptic, the combined system of Grad-Shafranov-Bernoulli equations can be hyperbolic in at least part of the solution domain in the presence of "transonic" poloidal flows (of the order of the sound speed $c_{s}$ times the ratio of poloidal magnetic field $B_{\theta}$ to total field $B$; throughout this paper, we will use the term transonic in this sense). Betti and Freidberg ${ }^{13}$ drew attention to a potentially important consequence of this for tokamak plasmas, namely, that spatial transitions from elliptic to hyperbolic behavior and back again would imply the presence of radial discontinuities in variables such as plasma density. This possibility is of particular interest in the tokamak context since, as noted previously, there is a wellestablished correlation between poloidal flows and transport barriers, which are characterized by steep gradients in temperature and density. Neither Betti and Freidberg ${ }^{13}$ nor Guazzotto and co-workers ${ }^{16,18}$ explicitly linked transonic 
poloidal flows with transport barriers, although a link between such flows and transitions to the high confinement $(\mathrm{H}-)$ mode of tokamak operation was proposed by Shaing and co-workers in the early $1990 \mathrm{~s}^{22}$ One would expect discontinuities in MHD equilibria to be replaced with smooth gradients (for example, on length scales of the order of the ion skin depth or Larmor radius) in two-fluid or kinetic models. ${ }^{17,23}$ Indeed it has been shown explicitly that no transition to hyperbolic behavior occurs at transonic values of the poloidal flow when either two-fluid ${ }^{17}$ or kinetic ${ }^{20}$ effects are taken into account. It is nevertheless tempting to believe that transport barriers in tokamaks can be identified with discontinuous solutions of the steady-state MHD equations, while recognizing that MHD by itself cannot provide a full understanding of transport barrier physics.

Our aim in this paper is to clarify some of the outstanding issues raised by the presence of equilibrium poloidal and toroidal flows in tokamak plasmas. In Sec. II, using results published previously in the astrophysical literature for the case of isentropic flux surfaces, we first recover an expression obtained by Goedbloed ${ }^{17}$ for the range of poloidal flow speeds for which the GS equation is hyperbolic, valid for arbitrary values of plasma beta $\beta$ and magnetic field pitch $B_{\theta} / B$. We then show that this result can be easily modified to describe the more tokamak-relevant scenario of isothermal flux surfaces and show explicitly that when $\beta$ and $B_{\theta} / B$ are of order unity (which could be realized in high performance spherical tokamak plasmas), the GS equation is hyperbolic for a significant range of poloidal flows. We obtain a simple expression for the variation of density on a flux surface when poloidal and toroidal flows are simultaneously present, and discuss how this expression could be exploited to infer information on poloidal and toroidal flows indirectly from experimental data. Finally, in this section, we demonstrate that there are four possible solutions for the plasma density when the flux surfaces are assumed to be isothermal, corresponding to four distinct poloidal flow regimes. In Sec. III, we discuss our analysis in the light of specific, published examples of poloidal flow measurements, and also predictions of poloidal flows based on neoclassical and turbulent transport modeling.

\section{CHARACTER OF GRAD-SHAFRANOV-BERNOULLI EQUATIONS}

\section{A. Isentropic flux surfaces}

Ideal MHD equilibria with flow $\mathbf{v}$ and isotropic scalar pressure $p$ are described by the steady-state equations of continuity and momentum

$$
\begin{aligned}
& \nabla \cdot(\rho \mathbf{v})=0, \\
& \rho(\mathbf{v} \cdot \nabla) \mathbf{v}=-\nabla p+\frac{1}{\mu_{0}}(\nabla \times \mathbf{B}) \times \mathbf{B},
\end{aligned}
$$

together with the ideal form of Ohm's law

$$
\mathbf{v} \times \mathbf{B}=\nabla \Phi,
$$

the Maxwell equation

$$
\nabla \cdot \mathbf{B}=0,
$$

and, to provide closure, an equation expressing either conservation of entropy per unit mass along streamlines

$$
\mathbf{v} \cdot \nabla\left(\frac{p}{\rho^{\gamma}}\right)=0
$$

or thermal equilibrium within magnetic flux surfaces

$$
\mathbf{B} \cdot \nabla\left(\frac{p}{\rho}\right)=0 .
$$

In Eqs. (1)-(6), $\rho$ is the mass density, $\mathbf{B}$ is the magnetic field, $\Phi$ is the electrostatic potential, $\mu_{0}$ is the free space permeability, and $\gamma$ is the ratio of specific heats. Lovelace and co-workers ${ }^{12}$ used these equations (with the addition of a gravitational force term in the momentum equation) to obtain Grad-Shafranov-Bernoulli equations describing axisymmetric MHD equilibria in the presence of arbitrary toroidal and poloidal flows, with Eqs. (5) and (6) both used as closure conditions. This analysis was later generalized by McClements and Thyagaraja ${ }^{14}$ to include two-fluid effects. If one uses the Bernoulli relation to express $\rho$ in terms of $\nabla \Psi$ where $\Psi$ is poloidal flux, defined such that the poloidal magnetic field is equal to $\nabla \Psi \times \nabla \varphi$ where $\varphi$ is toroidal angle, one can show that the terms in the GS equation involving second order derivatives of $\Psi$ can be written as ${ }^{12}$

$$
\left(1-\mu_{0} \frac{F^{\prime 2}}{\rho}\right)\left(A_{R R} \frac{\partial^{2} \Psi}{\partial R^{2}}+A_{R Z} \frac{\partial^{2} \Psi}{\partial R \partial Z}+A_{Z Z} \frac{\partial^{2} \Psi}{\partial Z^{2}}\right),
$$

where $R$ and $Z$ are, respectively, distance from the symmetry axis and distance along this axis, $F$ is defined such that the fluid momenta per unit volume in the $R$ and $Z$ directions are given by

$$
\begin{gathered}
\rho v_{R}=-\frac{1}{R} \frac{\partial F}{\partial Z}, \\
\rho v_{Z}=\frac{1}{R} \frac{\partial F}{\partial R},
\end{gathered}
$$

and, in the case of isentropic closure, the coefficients $A_{R R}$, $A_{R Z}$, and $A_{Z Z}$ can be written as

$$
\begin{aligned}
& A_{R R}=1-\frac{v_{\theta}^{2} v_{Z}^{2}}{c_{s}^{2} c_{A \theta}^{2}-\left(c_{s}^{2}+c_{A}^{2}\right) v_{\theta}^{2}+v_{\theta}^{4}}, \\
& A_{R Z}=\frac{2 v_{\theta}^{2} v_{R} v_{Z}}{c_{s}^{2} c_{A \theta}^{2}-\left(c_{s}^{2}+c_{A}^{2}\right) v_{\theta}^{2}+v_{\theta}^{4}}, \\
& A_{Z Z}=1-\frac{v_{\theta}^{2} v_{R}^{2}}{c_{s}^{2} c_{A \theta}^{2}-\left(c_{s}^{2}+c_{A}^{2}\right) v_{\theta}^{2}+v_{\theta}^{4}} .
\end{aligned}
$$

In the above expressions, $v_{\theta}=\left(v_{R}^{2}+v_{Z}^{2}\right)^{1 / 2}$ is the poloidal flow speed, $c_{s}=(\gamma p / \rho)^{1 / 2}$ is the adiabatic sound speed, $c_{A}=B /\left(\mu_{0} \rho\right)^{1 / 2}$ is the Alfvén speed, and $c_{A \theta}$ is the projection of the Alfvén speed onto the poloidal plane. The existence of a function $F$ satisfying Eqs. (8) and (9) arises from Eq. (1), applied to an axisymmetric plasma. It follows immediately from the toroidal component of Eq. (3), again under condi- 

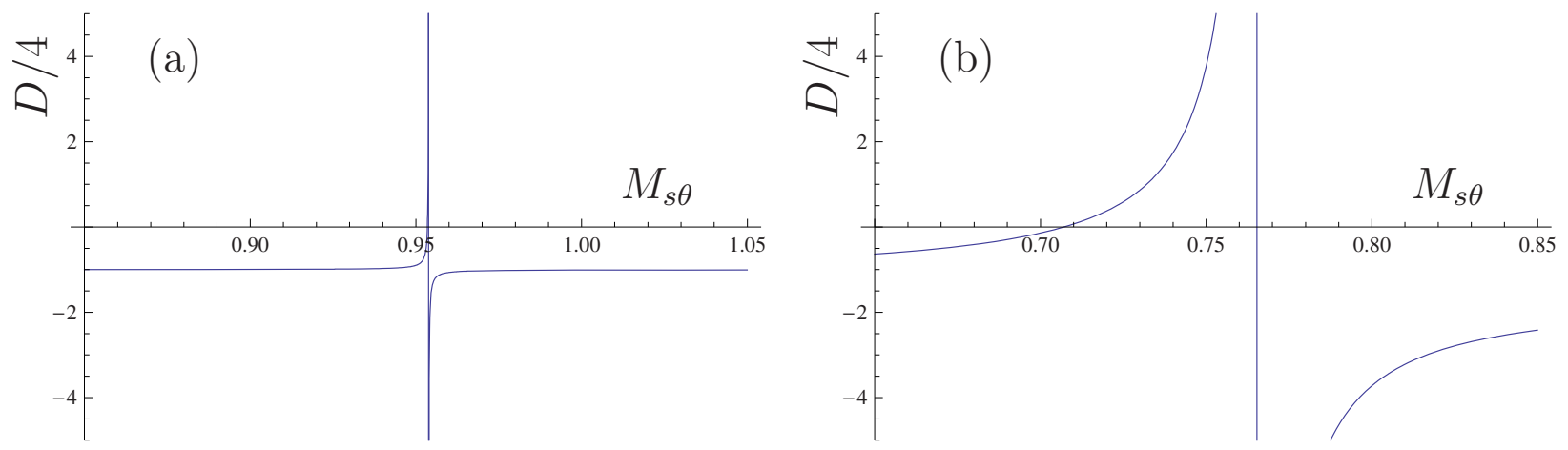

FIG. 1. (Color online) Discriminant $D$ of the second order derivatives in the Grad-Shafranov equation plotted vs poloidal sonic Mach number $M_{s \theta}$ for (a) $\beta=0.1, B_{\theta}=0.1 B$ and (b) $\beta=1, B_{\theta}=B_{\varphi}$. Negative (positive) values of $D$ indicate that the Grad-Shafranov equation is elliptic (hyperbolic).

tions of axisymmetry (i.e., $\partial \Phi / \partial \varphi=0$ ), that $F$ must be a flux function, ${ }^{14}$ i.e., $F=F(\Psi)$; in Eq. (7) $F^{\prime} \equiv d F / d \Psi$. It then follows from Eqs. (8) and (9) that $\rho v_{\theta}=F^{\prime} B_{\theta}$, where $B_{\theta}=|\nabla \Psi| / R$ is the absolute value of the poloidal magnetic field.

The GS equation is defined to be elliptic if $D \equiv A_{R Z}^{2}$ $-4 A_{R R} A_{Z Z}$ is negative, parabolic if $D=0$, and hyperbolic if $D>0 .{ }^{24}$ It is straightforward to establish that

$$
D=4 \frac{v_{\theta}^{2}\left(c_{s}^{2}+c_{A}^{2}\right)-c_{s}^{2} c_{A \theta}^{2}}{c_{s}^{2} c_{A \theta}^{2}-\left(c_{s}^{2}+c_{A}^{2}\right) v_{\theta}^{2}+v_{\theta}^{4}}
$$

In the absence of poloidal flows $\left(v_{\theta}=0\right)$, we obtain $D=-4$, indicating that the GS equation is elliptic in this limit. For the purpose of establishing the criteria for $D$ to change sign, it is useful to write Eq. (13) in terms of dimensionless variables. Following Guazzotto and co-workers, ${ }^{16}$ we define a poloidal sonic Mach number $M_{s \theta} \equiv\left(v_{\theta} / c_{s}\right)\left(B / B_{\theta}\right)$, where $B$ is the total magnetic field and a plasma beta $\beta=\gamma \mu_{0} p / B^{2}$. Equation (13) can then be written in the form

$$
D=-4 \frac{1-M_{s \theta}^{2}(1+\beta)}{1-M_{s \theta}^{2}(1+\beta)+\beta M_{s \theta}^{4}\left(B_{\theta} / B\right)^{2}} .
$$

Since $\beta M_{s \theta}^{4}\left(B_{\theta} / B\right)^{2}$ is positive definite it is apparent that the first change in the character of the equation as $v_{\theta}$ increases from zero occurs when $M_{s \theta}=1 /(1+\beta)^{1 / 2}$. At this critical Mach number, the equation is parabolic $(D=0)$; at slightly higher poloidal flows, it is hyperbolic $(D>0)$. The next transition, a reversion to elliptic behavior, occurs when the denominator in Eq. (14) vanishes. Since the denominator is quadratic in $M_{s \theta}^{2}$ it has two roots. The lower root, corresponding to the hyperbolic-elliptic transition, is given in general by ${ }^{17}$

$$
M_{s \theta}^{2}=\frac{(1+\beta)}{2 \beta} \frac{B^{2}}{B_{\theta}^{2}}\left[1-\left(1-\frac{4 \beta}{(1+\beta)^{2}} \frac{B_{\theta}^{2}}{B^{2}}\right)^{1 / 2}\right] .
$$

This result is valid for arbitrary plasma beta and magnetic field pitch $B_{\theta} / B$. Adopting the usual ordering for conventional tokamaks, i.e., assuming that $\beta B_{\theta}^{2} / B^{2} \ll 1$, and expanding the right hand side of Eq. (15) to second order in $4 \beta\left(B_{\theta} / B\right)^{2} /(1+\beta)^{2}$, we obtain

$$
M_{s \theta}^{2} \simeq \frac{1}{1+\beta}\left[1+\frac{\beta}{(1+\beta)^{2}} \frac{B_{\theta}^{2}}{B^{2}}\right] .
$$

Hence, if $\beta B_{\theta}^{2} / B^{2} \ll 1$, the GS equation is hyperbolic for poloidal sonic Mach numbers in the range

$$
\frac{1}{1+\beta}<M_{s \theta}^{2} \leqslant \frac{1}{1+\beta}\left[1+\frac{\beta}{(1+\beta)^{2}} \frac{B_{\theta}^{2}}{B^{2}}\right] .
$$

Equation (17) differs from the corresponding inequality quoted by Guazzotto and co-workers ${ }^{16}$ in that there is an erroneous factor of $1 / 4$ in the $B_{\theta}$-dependent term in the upper limit on $M_{s \theta}^{2}$ quoted by these authors, and hence, for a given set of plasma parameters, they underestimate the range of Mach numbers in which the GS equation is hyperbolic [see Eq. (23) in Ref. 16]. Guazzotto and co-workers used an elliptic code FLOW to solve numerically the GS equation for conventional tokamak plasmas with strong poloidal flows; density discontinuities were observed, as expected, in regions of the plasma where the poloidal flow Mach number exceeded the lower limit given by Eq. (17), i.e., $1 /(1+\beta)^{1 / 2}$ (see Fig. 11 in Ref. 16). The use of an elliptic code to compute solutions of an equation exhibiting hyperbolic behavior in part of the solution domain was justified a posteriori on the grounds that the hyperbolic regions were found to be of very limited extent, and consequently did not prevent the code from converging.

The ordering required for this conclusion to be valid, i.e., $\beta B_{\theta}^{2} / B^{2} \ll 1$, is not necessarily satisfied in the outboard region of spherical tokamak plasmas, where the poloidal and toroidal components of the magnetic field can be of comparable magnitude and the local plasma beta can be of order unity. In these circumstances, it is necessary to use Eq. (15) rather than Eq. (16) to compute the critical Mach number at which the GS equation reverts to being elliptic, and the range of Mach numbers for which the equation is hyperbolic can be considerably greater than it is in the high aspect ratio, low beta limit.

This point is illustrated by Fig. 1, in which $D / 4$ is plotted versus $M_{s \theta}$ for two pairs of values of $\beta$ and $B_{\theta} / B$, corresponding approximately to high performance regimes in (a) a conventional tokamak and (b) a spherical tokamak. It should be noted that the absolute values of $M_{s \theta}$ in the two plots are 
different, reflecting the fact that the threshold for the transition to hyperbolic behavior decreases with rising $\beta$. It is clear from the left hand plot that for low values of plasma beta and magnetic field pitch, the GS equation is hyperbolic for only a very narrow range of values of $M_{s \theta}$, as noted by Guazzotto and co-workers. ${ }^{16}$ If, on the other hand, $\beta=1$ and $B_{\theta}$ equals the toroidal field $B_{\varphi}$, as in the right hand plot of Fig. 1, we find that $D>0$ for a significant range of Mach numbers $0.7 \lessgtr M_{s \theta} \leq 0.76$. For a wide range of Mach numbers outside this interval, $D$ differs markedly from -4 , indicating significant poloidal flow effects on the equilibrium even at Mach numbers well below $1 /(1+\beta)^{1 / 2}$. This suggests that the presence of transonic poloidal flows in high performance spherical tokamak plasmas, with $\beta$ and $B_{\theta} / B_{\varphi}$ both of order unity, could pose serious numerical challenges for equilibrium reconstruction. For the conventional tokamak equilibria with transonic poloidal flows computed by Guazzotto and co-workers ${ }^{16}$ the hyperbolic region was so narrow in physical space that it could not be resolved using FLOW. It is far from clear that elliptic codes of this type could be used to compute transonic equilibria when $\beta$ and $B_{\theta} / B_{\varphi}$ are of order unity. Indeed, it is not clear that MHD equilibrium solutions even exist in this regime, although it should always be possible to recover transonic equilibria by introducing two-fluid or kinetic effects. ${ }^{17,20}$

\section{B. Isothermal flux surfaces}

The above analysis is based on the assumption that entropy per unit mass is a flux function. If we assume in addition that temperature is a flux function, on the reasonable physical grounds that parallel heat transport in tokamak plasmas is extremely rapid, then $\rho$ must also be a flux surface quantity. However, a two-fluid analysis of dissipationless equilibrium force balance in the presence of toroidal flows indicates that $\rho$ cannot be a flux function ${ }^{1}$ and, moreover, it can be shown that equilibrium poloidal flows cannot occur in the absence of toroidal flows. ${ }^{16,25}$ When the flow is purely toroidal and a single ion species is present, it is straightforward to show that ideal MHD and dissipationless two-fluid theory lead to the same result for the variation of density on rigidly rotating isothermal flux surfaces, namely, $\exp \left[m_{i} \omega^{2} R^{2} / 4 T\right]$, where $m_{i}$ is the ion mass, $\omega$ is the toroidal rotation rate, and $T \simeq m_{i} p /(2 \rho)$ is the arithmetic mean of the ion and electron temperatures ${ }^{19}$ (it should be noted, however, that whereas ideal MHD requires $\Phi$ to be flux function, twofluid theory requires this quantity to vary on a flux surface when $\omega \neq 0$ ). Thomson scattering measurements of midplane density profiles in spherical tokamak plasmas with rapid toroidal rotation appear to be broadly consistent with this result. ${ }^{26}$ For the purpose of modeling the effects on plasma equilibria of poloidal flows, one may conclude that there are both experimental and theoretical grounds for assuming that flux surfaces in tokamaks are more nearly isothermal than isentropic.

It is straightforward to adapt the analysis to accommodate isothermal flux surfaces. Specifically, it is necessary to derive a modified Bernoulli relation from the parallel component of Eq. (2), the steady-state ideal MHD momentum equation. Using Eq. (3), it is straightforward to show that electrostatic potential $\Phi$ is a flux function with derivative ${ }^{14}$

$$
\Omega \equiv \Phi^{\prime}=\frac{v_{\varphi}}{R}-\frac{B_{\varphi} F^{\prime}}{\rho R},
$$

where $v_{\varphi}$ is the toroidal flow speed. The Bernoulli relation for the case of isentropic flux surfaces can then be written in the form ${ }^{14}$

$$
H_{1}(\Psi)=\frac{\gamma}{\gamma-1} \sigma \rho^{\gamma-1}+\frac{v_{\varphi}^{2}+v_{\theta}^{2}}{2}-\Omega R v_{\varphi}
$$

where $H_{1}$ and $\sigma$ are flux functions. If, on the other hand, $T$ is assumed to be a flux function, $H_{1}$ is replaced with a flux function

$$
H_{2}(\Psi)=\frac{2 T}{m_{i}} \ln \left(\frac{\rho}{\rho_{0}}\right)+\frac{v_{\varphi}^{2}+v_{\theta}^{2}}{2}-\Omega R v_{\varphi},
$$

where $\rho_{0}$ is an arbitrary constant density. Equation (20) can be obtained directly from Eq. (19) by taking the limit $\gamma \rightarrow 1$, noting that $\left(x^{\epsilon}-1\right) / \epsilon \rightarrow \ln x$ for $x>0$ as $\epsilon \rightarrow 0$.

The possibility of the GS equation exhibiting hyperbolic behavior arises from the fact that all of the second order derivatives in the equation are contained in the following terms:

$$
\begin{aligned}
R \frac{\partial}{\partial R}\left(\frac{\chi}{R} \frac{\partial \Psi}{\partial R}\right)+\frac{\partial}{\partial Z}\left(\chi \frac{\partial \Psi}{\partial Z}\right)= & \chi\left\{R \frac{\partial}{\partial R}\left(\frac{1}{R} \frac{\partial \Psi}{\partial R}\right)+\frac{\partial^{2} \Psi}{\partial Z^{2}}\right\} \\
& +\nabla \chi \cdot \nabla \Psi
\end{aligned}
$$

where

$$
\chi=1-\mu_{0} \frac{F^{\prime 2}}{\rho} .
$$

Equations (19) and (20) provide relations between $\rho$ and the first order derivatives of $\Psi$, which appear in $v_{\theta}$ by virtue of Eqs. (8) and (9) and the fact that $F$ is a flux function. It follows that $\nabla \rho$, which appears in the GS equation via the $\rho$ dependence of $\chi$, can be expressed in terms of second order derivatives of $\Psi$. The required relation can be obtained by taking the gradient of Eq. (19) or Eq. (20), depending on whether specific entropy or temperature is assumed to be a flux function. We note that

$$
\nabla\left[\frac{\gamma}{\gamma-1} \sigma \rho^{\gamma-1}\right]=\frac{\gamma p}{\rho} \frac{\nabla \rho}{\rho}+\frac{\gamma \rho^{\gamma-1}}{\gamma-1} \sigma^{\prime} \nabla \Psi
$$

and

$$
\nabla\left[\frac{2 T}{m_{i}} \ln \left(\frac{\rho}{\rho_{0}}\right)\right]=\frac{2 T}{m_{i}} \frac{\nabla \rho}{\rho}+\frac{2 \ln \left(\rho / \rho_{0}\right)}{m_{i}} T^{\prime} \nabla \Psi .
$$

As far as the dependences of $\nabla \rho$ on the second order derivatives of $\Psi$ are concerned, it is clear from these expressions that the sole difference between the two closure conditions is that $c_{s}^{2} \equiv \gamma p / \rho$ in the adiabatic case is replaced with $v_{i}^{2} \equiv 2 T / m_{i}$ in the isothermal case. Thus, Eqs. (14)-(17) remain applicable with the plasma beta redefined as $\beta=\mu_{0} p / B^{2}$ and the poloidal sonic Mach number redefined as $M_{s \theta}=\left(v_{\theta} / v_{i}\right)\left(B / B_{\theta}\right)$. Since $\gamma \simeq 5 / 3$ for a fully ionized plasma and $2 T / m_{i} \simeq p / \rho$, it follows from Eq. (17) that when $\beta \ll 1$, 
the threshold poloidal flow for the GS equation to become hyperbolic is a factor of $(5 / 3)^{1 / 2} \simeq 1.3$ lower in the isothermal case than it is in the adiabatic case. In view of the fact that tokamak flux surfaces are likely to be more nearly isothermal than isentropic, this reduction in the threshold poloidal flow for radially discontinuous solutions to occur could have significant implications for the interpretation of experimental data.

It is apparent from Eqs. (8), (9), and (18) that

$$
\Omega(\Psi)=\frac{v_{\varphi}}{R}-\frac{v_{\theta}}{R} \frac{B_{\varphi}}{B_{\theta}} .
$$

When this expression is substituted into the Bernoulli relation for isothermal flux surfaces [Eq. (20)], it is apparent that we can formally write

$$
n=n_{1}(\Psi) \exp \left[\frac{v_{\varphi}^{2}}{2 v_{i}^{2}}-\frac{v_{\theta}^{2}}{2 v_{i}^{2}}-\frac{v_{\varphi} v_{\theta}}{v_{i}^{2}} \frac{B_{\varphi}}{B_{\theta}}\right],
$$

where $n \simeq \rho / m_{i}$ is the electron number density and $n_{1}=\left(\rho_{0} / m_{i}\right) \exp \left[H_{2}(\Psi) / v_{i}^{2}\right]$ is a flux function. It should be stressed that this result does not represent an exact solution for the density in terms of flux surface quantities and space variables, since $v_{\theta}=F^{\prime} B_{\theta} / \rho$ and $v_{\varphi}$ can also be expressed in terms of $\rho$. Equation (26) may nevertheless be regarded as a formal generalization to the finite $v_{\theta}$ case of the expression first obtained by Hinton and Wong ${ }^{1}$ for the variation of density in a plasma with purely toroidal rotation.

Eliminating $v_{\varphi}$ from Eq. (26) using Eq. (25) and defining

$$
n_{2}(\Psi)=n_{1}(\Psi) \exp \left[\frac{\Omega^{2} R_{0}^{2}}{2 v_{i}^{2}}\right],
$$

where the constant $R_{0}$ may be conveniently chosen to be the magnetic axis location, we obtain

$$
n=n_{2}(\Psi) \exp \left[\frac{\Omega^{2}(\Psi)}{2 v_{i}^{2}(\Psi)}\left(R^{2}-R_{0}^{2}\right)-\frac{1}{2} M_{s \theta}^{2}\right] .
$$

Since the poloidal flow must vanish at the magnetic axis, the flux function $n_{2}=n$ at this point. Equation (28) could be used to obtain experimental information on poloidal flows in tokamaks, as follows. Thomson scattering measurements are routinely used to determine high resolution electron density and temperature $\left(T_{e}\right)$ profiles across the entire plasma midplane. Moreover, Doppler shifts and widths of impurity spectral emission lines can be used to measure toroidal/poloidal flows and ion temperatures $T_{i}$, while motional Stark effect (MSE) measurements yield the pitch of the magnetic field, $B_{\theta} / B_{\varphi}$. However, unlike Thomson scattering measurements of $n$ and $T_{e}$, it is generally not possible to obtain experimental profiles of $v_{\varphi}, v_{\theta}, T_{i}$, or $B_{\theta} / B_{\varphi}$ on the high field side of the core plasma. The reason for this is that the diagnostics used to obtain measurements of core plasma flows, ion temperature, and magnetic field pitch rely on the presence of neutral beams to provide electrons for charge exchange and high neutral particle velocities for the motional Stark effect. Because of beam attenuation (and also, in some cases, the fact that the beam line is tangential to a flux surface that lies close to the magnetic axis), neutral beams cannot generally be used to obtain diagnostic information in the innermost regions of the plasma, although gas puffing can be used to provide flow measurements close to the last closed flux surface on the high field side. ${ }^{27}$

Equation (28) can, in principle, help to remedy this difficulty. All of the quantities on the right hand side of Eq. (25) and in $M_{s \theta}$ are measurable at a particular $R$ on the low field side of the plasma. If we make the reasonable assumption that electron temperature is a flux function, Thomson scattering measurements can then be used to determine the corresponding point on the flux surface on the high field side. The inboard/outboard density asymmetry, which can also be measured with high accuracy using Thomson scattering, then enables us to determine $M_{s \theta}$ on the high field side. While it may not be possible to obtain $v_{\theta}$ or $B_{\theta}$ individually on the high field side, it is the poloidal sonic Mach number that determines whether the GS equation is elliptic or hyperbolic, and hence whether density discontinuities of the type discussed by Guazzotto and co-workers ${ }^{16}$ are present in the plasma.

We note also that Eq. (25) can be rearranged to give

$$
v_{\varphi}=R \Omega(\Psi)+v_{i}(\Psi) M_{s \theta}\left(1-\frac{B_{\theta}^{2}}{B^{2}}\right)^{1 / 2} .
$$

In the limit $B_{\theta}^{2} \ll B^{2}$, which is generally valid on the high field side of both conventional and spherical tokamaks, this expression reduces to

$$
v_{\varphi} \simeq R \Omega(\Psi)+v_{i}(\Psi) M_{s \theta} .
$$

Having used Eq. (28) to determine $M_{s \theta}$ on the high field side, we can then use Eq. (30) to obtain an approximate value of $v_{\varphi}$ at the same location. We would thereby obtain a more complete picture of the global dynamics of tokamak plasmas with flows than is possible with existing diagnostics.

It should be noted that the method proposed above does not require any assumptions to be made regarding the plasma equilibrium, apart from the usual assumption of axisymmetry and the physically reasonable assumption of isothermal flux surfaces. It does not therefore require us to make any a priori assumptions regarding the presence or otherwise of hyperbolic regions in the solution domain of the GS equation. One caveat that should be made here is that the inference of magnetic field pitch from MSE measurements is complicated by the presence of any laboratory frame electric fields, including those associated with plasma rotation. It is possible to correct the MSE measurements for the effect of any such fields by measuring simultaneously $v_{\varphi}$ and $v_{\theta}$; these measurements are in any case required on the low field side in order for the method to be viable. Another caveat is that we assumed in our analysis that only a single ion species contributes significantly to the mass density. However, the method could also be applied to a plasma with a single, dominant impurity species, provided that visible bremsstrahlung measurements of effective charge could be made across the plasma midplane to enable total mass density to be calculated from Thomson scattering measurements of electron number density.

While Eq. (28) could be used to infer useful experimental information on poloidal and toroidal flows, as noted previously it does not provide a true solution for $n$ in terms of 
flux functions and space variables since $M_{s \theta}$ is itself a function of $n$. Although the isothermal Bernoulli relation Eq. (20) cannot in fact be solved exactly for $n$, we can determine the number of real roots of this equation for any given set of parameters, and it is important to do so since, as noted by Betti and Freidberg, ${ }^{13}$ it is the existence of more than one solution for the density that leads to the possibility of radial discontinuities.

The poloidal sonic Mach number in Eq. (28) can be written as

$$
M_{s \theta}=\frac{v_{\theta}}{v_{i}} \frac{B}{B_{\theta}}=\frac{F^{\prime}}{\rho v_{i}}\left(B_{\theta}^{2}+B_{\varphi}^{2}\right)^{1 / 2},
$$

and it is straightforward to show that there exists a flux function $f$ such that ${ }^{19}$

$$
f(\Psi)=R B_{\varphi}-\mu_{0} F^{\prime} R v_{\varphi} .
$$

Equations (18) and (32) can be combined to express the toroidal field in terms of the density

$$
B_{\varphi}=\frac{1}{R} \frac{f+\mu_{0} F^{\prime} \Omega R^{2}}{1-\mu_{0} F^{\prime 2} / \rho} .
$$

Using this result in Eq. (31) and substituting the resulting expression into Eq. (28), we obtain

$$
\begin{aligned}
n= & n_{2}(\Psi) \exp \left[\frac{\Omega^{2}(\Psi)}{2 v_{i}^{2}(\Psi)}\left(R^{2}-R_{0}^{2}\right)-\frac{F^{\prime 2}}{2 m_{i}^{2} v_{i}^{2} n^{2}}\right. \\
& \left.\times\left\{B_{\theta}^{2}+\left(\frac{f / R+\Omega R \mu_{0} F^{\prime}}{1-\mu_{0} F^{\prime 2} /\left(m_{i} n\right)}\right)^{2}\right\}\right] .
\end{aligned}
$$

For the purpose of discussing the roots $n=n(\Psi, R, Z)$ of this equation, it is convenient to introduce a normalized density $x=n / n_{0}$, where $n_{0}$ is an arbitrary constant density, a sonic Mach number $M_{s}=F^{\prime} B_{\theta} /\left(m_{i} n_{0} v_{i}\right)$, a poloidal Alfvén Mach number $M_{A \theta}=F^{\prime}\left(\mu_{0} / m_{i} n_{0}\right)^{1 / 2}$, a magnetic field pitch parameter

$$
\xi=\frac{1}{B_{\theta}}\left(\frac{f}{R}+\Omega R \mu_{0} F^{\prime}\right),
$$

and a dimensionless spatially varying density

$$
x_{0}=\frac{n_{2}(\Psi)}{n_{0}} \exp \left[\frac{\Omega^{2}}{2 v_{i}^{2}}\left(R^{2}-R_{0}^{2}\right)\right] .
$$

Equation (34) can then be written in the form

$$
x=x_{0} \exp \left[-\frac{M_{s}^{2}}{2 x^{2}}\left\{1+\frac{\xi^{2} x^{2}}{\left(x-M_{A \theta}^{2}\right)^{2}}\right\}\right] .
$$

The real roots of this equation, and whether any such roots exist, can be determined graphically by plotting the functions

$$
g_{1}(x)=\exp \left[-\frac{M_{s}^{2}}{2 x^{2}}\left\{1+\frac{\xi^{2} x^{2}}{\left(x-M_{A \theta}^{2}\right)^{2}}\right\}\right],
$$

and $g_{2}(x)=x / x_{0}$ for prescribed values of $M_{s}, M_{A \theta}$, $\xi$, and $x_{0}$. Figure 2 shows $g_{1}$ for $M_{s}=0.1, M_{A \theta}=0.1$, and $\xi=10$, all of which are realistic tokamak values, together with $g_{2}$ for a range of values of $x_{0}$. It is evident that there are two roots when $x_{0}$ exceeds some critical value, which is approximately

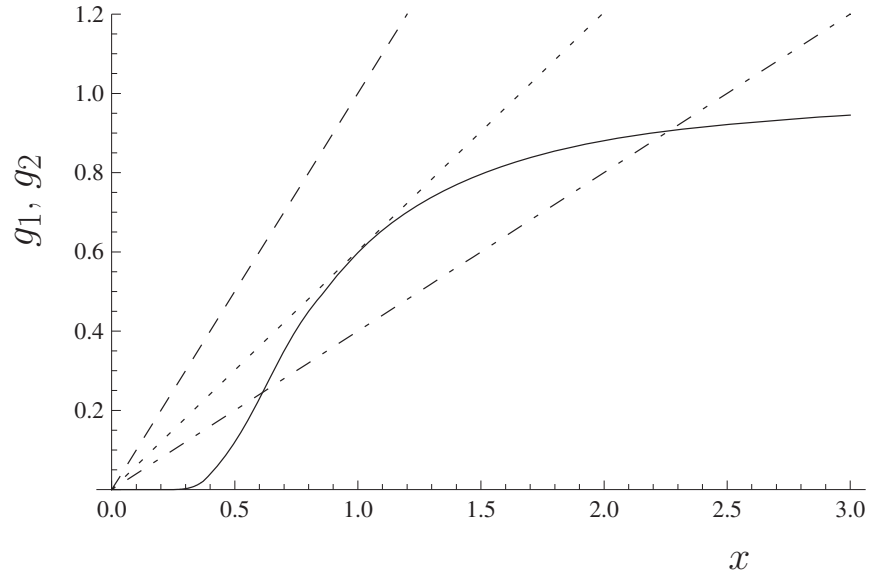

FIG. 2. The functions $g_{1}(x)$ for $M_{s}=0.1, M_{A \theta}=0.1, \xi=10$ (solid curve), and $g_{2}(x)$ for $x_{0}=1.0$ (dashed line), 1.65 (dotted line), and 2.5 (dashed-dotted line). Solutions $n=n_{0} x$ of the Bernoulli relation correspond to $g_{2}=g_{1}$, i.e., intersections of the solid curve with the straight lines.

1.65 in the case of the other parameter values assumed in Fig. 2; for $x_{0}$ less than this critical value, no solutions exist. Betti and Freidberg ${ }^{13}$ showed analytically that poloidal flows in a low $\beta$, large aspect ratio tokamak with isentropic flux surfaces lead to high and low density solutions, and consequently the possibility of density discontinuities. The above analysis and Fig. 2 suggest that this result applies generally to tokamaks with arbitrary $\beta$ and aspect ratio, and isothermal flux surfaces. In the absence of poloidal flow, there is only one solution for the density; this can be seen by setting $M_{s \theta}=0$ in Eq. (28).

The critical value of $x_{0}$ required for two coincident roots of Eq. (37) to exist, and the physical significance of this value can be deduced by recognizing first that poloidal flows in tokamaks are generally much smaller than the poloidal Alfvén speed, i.e., $M_{A \theta} \ll 1$, while in conventional tokamaks, $\xi \simeq B_{\varphi} / B_{\theta} \gg 1$. If we assume, moreover, that $x$ is of order unity, Eq. (38) then reduces to

$$
g_{1}(x) \simeq \exp \left[-\frac{M_{s}^{2} \xi^{2}}{2 x^{2}}\right] \text {. }
$$

The conditions for two coincident roots of Eq. (37) to exist are that $g_{1}=g_{2}$ and $g_{1}^{\prime}=g_{2}^{\prime}$. In the limit in which Eq. (39) is applicable, it is straightforward to show that this requires $x=M_{s} \xi$ and $x_{0}=M_{s} \xi e^{1 / 2} \simeq 1.65$ when $M_{s}=0.1, \xi=10$ as in Fig. 2 . We then have $M_{s \theta}=M_{s} B /\left(B_{\theta} x\right) \simeq M_{s} \xi / x=1$, i.e., when $x_{0}$ is equal to its critical value the poloidal flow is transonic. When $x_{0}$ is greater than this critical value, there are two nondegenerate solutions for the density, corresponding to subsonic $\left(x>M_{s} \xi\right)$ and supersonic $\left(x<M_{s} \xi\right)$ poloidal flow.

Other solutions of Eq. (37) become apparent if we do not assume $M_{s} \ll 1, M_{A \theta} \ll 1$, and $\xi \gg 1$. It follows from the definitions of these quantities that $M_{A \theta} \sim M_{s} \xi \beta^{1 / 2}$ where $\xi \sim B_{\varphi} / B_{\theta}$. Since, as noted previously, $\beta$ and $B_{\theta} / B_{\varphi}$ can both be of order unity in a spherical tokamak, it is of interest to plot $g_{1}(x)$ for $M_{s}=M_{A \theta}=\xi=1$; this is shown by the solid curve in Fig. 3, along with $g_{2}(x)$ (the dashed-dotted line) for $x_{0}=40$. The function $g_{1}$ now has a local maximum at $x=0.5$, implying the existence of four roots of Eq. (37) when 


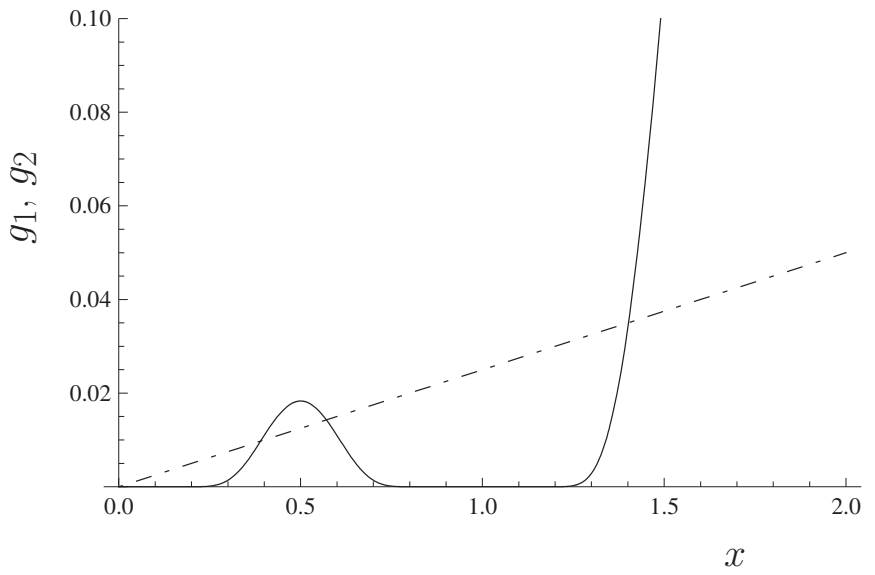

FIG. 3. The functions $g_{1}(x)$ for $M_{s}=1, M_{A \theta}=1$, and $\xi=1$ (solid curve) and $g_{2}(x)$ for $x_{0}=40$ (dashed-dotted line).

$x_{0}$ is sufficiently large. Three of these roots are indicated in Fig. 3; the fourth, which is not shown in the figure, arises from the fact that $g_{1} \rightarrow 1$ as $x \rightarrow \infty$, whereas $g_{2}$ is unbounded. Indeed for any set of values of $M_{s}, M_{A \theta}$, and $\xi$, there exist values of $x_{0}$ such that there are four density solutions. It is straightforward to show that the local maximum in $g_{1}$ occurs at

$$
x=\frac{M_{A \theta}}{1+\xi^{2 / 3}}
$$

and Fig. 3 shows that this lies between the two lowest density solutions. The poloidal flow speed corresponding to the normalized density given by Eq. (40) is

$$
v_{\theta}=c_{A \theta}\left(1+\xi^{2 / 3}\right) \text {. }
$$

Since $\xi \gtrsim 1$ in tokamaks, generally, and $\xi \gg 1$ in conventional tokamaks, it is apparent from Eq. (41) that the lowest density solutions are only applicable in the presence of poloidal flows that are comparable to or in excess of the poloidal Alfvén speed. The effects of such flows on tokamak equilibria with isentropic flux surfaces have been examined by Guazzotto and Betti. ${ }^{18}$ We will not pursue this possibility here, but merely note the theoretical possibility of density solutions corresponding to trans-Alfvénic flows in equilibria with isothermal flux surfaces. Such flows exceed transonic ones by a factor of order $\beta^{-1 / 2}$, which is much larger than unity in conventional tokamaks. In Sec. III, we will assess the experimental and transport theoretical evidence for the presence of transonic and supersonic flows in real tokamak plasmas.

\section{DISCUSSION AND CONCLUSIONS}

In the light of the analysis in Sec. II, it is worth considering whether there is experimental evidence for radial discontinuities of the type discussed by Guazzotto and co-workers. ${ }^{16}$ As noted previously, rapid poloidal rotation has been observed to be correlated with internal transport barriers in several tokamaks, including JET. ${ }^{10,28}$ The highest poloidal velocities recorded in this device, $75 \pm 20 \mathrm{~km} \mathrm{~s}^{-1}$ (in the ion diamagnetic direction), were measured inside a transport barrier in shot number 58094; immediately outside the barrier, the poloidal velocity measurement was consistent with zero. Between these two radial locations, respectively, at $R \simeq 3.34 \mathrm{~m}$ and $R \simeq 3.46 \mathrm{~m}$, the ion temperature was observed to drop from about 20 to about $7 \mathrm{keV}$, while the electron density dropped by approximately a factor of two, the local safety factor $q$ being close to 2 at both locations. ${ }^{28}$ These figures, combined with the magnetic axis location in JET $\left(R_{0} \simeq 3.0 \mathrm{~m}\right)$, suggest that the poloidal sonic Mach number was close to unity inside the barrier, but decreased sharply with increasing minor radius across the barrier, to a value close to zero. Abrupt falls in density and pressure were thus accompanied by a fall in Mach number.

In contrast, equilibrium solutions of the GS equation with poloidal and toroidal flows, computed by Guazzotto and co-workers, ${ }^{16}$ show discontinuous drops in density associated with rises in $M_{s \theta}$, from clearly subsonic to clearly supersonic values. This type of relation between $n$ and $M_{s \theta}$ was obtained for the particular case of isentropic flux surfaces; Eq. (28) indicates that it also holds in the isothermal flux surface case if the flux functions in this equation $\left(n_{2}, \Omega\right.$, and $v_{i}$ ) are continuous across the barrier. The fact that there is no indication in the JET data of clearly supersonic flows $\left(M_{s \theta} \gg 1\right)$, combined with the fact that the sign of the change in $M_{s \theta}$ across the barrier is the same as that of $n$, leads us to conclude that there is no evidence in these data of radial discontinuities of the type predicted by Betti and co-workers, ${ }^{13,16}$ although we emphasize again that these authors did not explicitly link such radial discontinuities with transport barriers. On the other hand, the fact that $M_{s \theta}$ was of order unity inside the barrier and close to zero outside it suggests that tokamak plasma density profiles can, in some circumstances, be significantly affected by poloidal flows [cf. Eq. (28)].

Poloidal flows measured in other tokamaks have generally been found to be smaller than those reported in Ref. 10 . In the Mega Ampère Spherical Tokamak (MAST), for example, $\left|v_{\theta}\right|$ is typically a few kilometers per second. ${ }^{29}$ In the case of H-mode MAST plasmas, the poloidal sonic Mach number remains well below unity throughout the pedestal region (cf. Fig. 7 in Ref. 29; note that $B_{\theta} / B_{\varphi}$ is generally close to unity in the outer midplane of MAST). Thus, the conclusion that there is no evidence in the JET data of transonic discontinuities also applies a fortiori to the MAST data, and therefore one should not interpret the transport barriers observed in these plasmas in such terms. It should also be noted that such barriers, by their very nature, are associated with large pressure gradients as well as substantial plasma flows, and the ion pressure gradient often makes a significant contribution to momentum balance in the ion fluid, thereby violating the ideal MHD Ohm's law [Eq. (3)] upon which the calculations by Betti and co-workers ${ }^{13,16}$ are based.

It is important to recognize that the above conclusion applies only to regions of the JET and MAST plasmas in which direct measurements of $v_{\theta}$ are currently possible, i.e., the outer midplane and the immediate vicinity of the magnetic axis. It does not exclude the possibility of radial discontinuities and supersonic flows on the inboard side, the 
existence of which could, in principle, be inferred indirectly using the method described in Sec. II, although Betti and Freidberg ${ }^{13}$ have argued (on the basis of a low beta, large aspect ratio expansion) that smooth transitions from subsonic to supersonic flow can occur in the inner midplane.

In evaluating the likelihood of supersonic poloidal flows occurring in tokamaks, the predictions of neoclassical and turbulent transport theory should also be considered. In a plasma with purely neoclassical transport, the bulk ions have been shown to have a poloidal flow, driven by the ion temperature gradient, of the order of $v_{i} \rho_{i} / L_{T i}$, where $\rho_{i}$ is the ion thermal Larmor radius and $L_{T i}$ is the ion temperature gradient length scale. ${ }^{30}$ This implies a poloidal sonic Mach number of order $\left(\rho_{i} / L_{T i}\right)\left(B / B_{\theta}\right)$, which could be close to unity in the vicinity of transport barriers, where $L_{T i}$ is much smaller than the plasma minor radius, although we again emphasize that the ideal MHD form of Ohm's law is not strictly applicable in these circumstances. Indeed, ideal MHD cannot be consistent with neoclassical poloidal flows since the latter are essentially diamagnetic drifts. Our analysis is probably not applicable to recent observations of impurity accumulation close to the inboard plasma edge in the Alcator C-Mod tokamak, since this appears to be a purely neoclassical effect. ${ }^{27}$ However, poloidal flows significantly exceeding in magnitude the predictions of neoclassical theory have been observed in JET (Ref. 10) and DIII-D (Ref. 31); in both cases, even the sign of the measured rotation is often at variance with neoclassical predictions. First principles-based fluid simulations of JET and Rijnhuizen tokamak project plasmas with internal transport barriers, carried out using the global electromagnetic turbulence code CUTIE, also reveal poloidal $\mathbf{E} \times \mathbf{B}$ velocities exceeding neoclassical predictions and, in the case of the JET simulations, having the same sign and approximately the same magnitude as the measured values. ${ }^{28,32}$ Careful examination of the simulation results has indicated that the flows are driven primarily by the Reynolds stress term in the ion fluid momentum equation. ${ }^{28}$

Notwithstanding the experimental and theoretical evidence for poloidal flows exceeding neoclassical values, the fact remains that no clear evidence has yet emerged in any tokamak plasma of flows with poloidal sonic Mach number exceeding unity, as envisaged in Refs. 13, 16, and 18. If such flows have indeed not occurred in any tokamak plasma, this could be due to the poloidal momentum sources in existing devices simply not being strong enough to break the poloidal sound barrier. Alternatively, it could imply that solutions of the GS equation satisfying the required boundary conditions do not in fact exist when there is a hyperbolic region in the solution domain, i.e., the presence of supersonic flows could be incompatible with equilibrium solutions of the ideal MHD equations. The latter possibility appears to be excluded by the fact that Guazzotto and co-workers were able to obtain convergent numerical solutions of the GS equation with coexisting regions of subsonic and supersonic poloidal flow, although they only demonstrated convergence in the limit $\beta B_{\theta}^{2} / B^{2} \ll 1$ (Ref. 16); it is not clear that stable equilibria exist in the case of the high performance spherical tokamak regime represented by Fig. 1(b). Nevertheless, it seems possible that the supersonic regime could be accessed in future experiments, with potentially significant consequences for the creation of transport barriers.

Our conclusions are as follows. We have demonstrated that a criterion for the GS equation to become elliptic, derived previously for the case of isentropic flux surfaces, can be easily modified to accommodate the more tokamakrelevant scenario of isothermal flux surfaces; the threshold poloidal flow for hyperbolicity to occur is slightly reduced as a result of this modification. It has also been shown that the range of transonic flows (of the order of the isothermal sound speed $v_{i}$ multiplied by the ratio of poloidal magnetic field $B_{\theta}$ to total field $B$ ) for which the GS equation is hyperbolic can be significantly greater in high performance spherical tokamak plasmas than in conventional tokamaks. We have shown that a simple expression for the variation of density on an isothermal flux surface in the presence of toroidal flows, obtained from the Bernoulli relation, can be generalized to include poloidal flows, and thereby used to infer experimental information about both types of flow from regions of the plasma where direct velocity measurements are not possible. We have shown graphically that the isothermal Bernoulli relation, if regarded as an algebraic equation for the plasma density, has, in general, four solutions in the presence of poloidal flows, rather than a single solution in the absence of such flows. These density solutions corresponds to four distinct poloidal flow regimes, namely, subsonic (with respect to $\left.v_{i} B_{\theta} / B\right)$, supersonic, sub-Alfvénic [with respect to a modified Alfvén speed defined by the right hand side of Eq. (41)] and super-Alfvénic. Although there is no clear experimental or transport-theoretical evidence of any regime other than the subsonic one being realized in tokamak plasmas, the possibility of accessing the other regimes in future experiments cannot at present be ruled out.

\section{ACKNOWLEDGMENTS}

This work was partly funded by the United Kingdom Engineering and Physical Sciences Research Council under Grant No. EP/G003955 and the European Communities under the contract of Association between EURATOM and CCFE. The views and opinions expressed herein do not necessarily reflect those of the European Commission. We thank A. Cooper (Centre de Recherches en Physique des Plasmas, École Polytechnique Fédérale de Lausanne), K. Crombé (Laboratory for Plasma Physics, École Royale Militaire, Brussels), A. Field, N. Hawkes, J. McCone, and A. Thyagaraja (CCFE) for helpful discussions.

\footnotetext{
${ }^{1}$ F. L. Hinton and S. K. Wong, Phys. Fluids 28, 3082 (1985).

${ }^{2}$ P. Helander, Phys. Plasmas 5, 1209 (1998).

${ }^{3}$ K. G. McClements and R. J. McKay, Plasma Phys. Controlled Fusion 51, 115009 (2009).

${ }^{4}$ M. Artun and W. M. Tang, Phys. Fluids B 4, 1102 (1992).

${ }^{5}$ K. H. Burrell, Phys. Plasmas 6, 4418 (1999).

${ }^{6}$ Y. Camenen, A. G. Peeters, C. Angioni, F. J. Casson, W. A. Hornsby, A. P. Snodin, and D. Strintzi, Phys. Plasmas 16, 012503 (2009).

${ }^{7}$ I. T. Chapman, T. C. Hender, S. Saarelma, S. E. Sharapov, R. J. Akers, and N. J. Conway, and MAST Team, Nucl. Fusion 46, 1009 (2006).

${ }^{8}$ A. M. Popov, in Proceedings of the 33rd EPS Conference on Plasma Physics, edited by F. De Marco and G. Vlad (European Physical Society, Rome, 2006), Vol. 30I, P-1.106.

${ }^{9}$ Y. Q. Liu, M. S. Chu, A. M. Garofalo, R. J. La Haye, Y. Gribov, M.
} 
Gryaznevich, T. C. Hender, D. F. Howell, P. de Vries, M. Okabayashi, S. D. Pinches, H. Reimerdes, and EFDA-JET Contributors, Phys. Plasmas 13, 056120 (2006).

${ }^{10}$ K. Crombé, Y. Andrew, M. Brix, C. Giroud, S. Hacquin, N. C. Hawkes, A. Murari, M. F. F. Nave, J. Ongena, V. Parail, G. Van Oost, I. Voitsekhovitch, and K.-D. Zastrow, Phys. Rev. Lett. 95, 155003 (2005).

${ }^{11}$ E. Hameiri, Phys. Fluids 26, 230 (1983).

${ }^{12}$ R. V. E. Lovelace, C. Mehanian, C. M. Mobarry, and M. E. Sulkanen, Astrophys. J., Suppl. Ser. 62, 1 (1986).

${ }^{13}$ R. Betti and J. P. Freidberg, Phys. Plasmas 7, 2439 (2000).

${ }^{14}$ K. G. McClements and A. Thyagaraja, Mon. Not. R. Astron. Soc. 323, 733 (2001).

${ }^{15}$ J. P. Goedbloed, A. J. C. Beliën, B. van der Holst, and R. Keppens, Phys. Plasmas 11, 28 (2004).

${ }^{16}$ L. Guazzotto, R. Betti, J. Manickam, and S. Kaye, Phys. Plasmas 11, 604 (2004).

${ }^{17}$ J. P. Goedbloed, Phys. Plasmas 11, L81 (2004).

${ }^{18}$ L. Guazzotto and R. Betti, Phys. Plasmas 12, 056107 (2005).

${ }^{19}$ A. Thyagaraja and K. G. McClements, Phys. Plasmas 13, 062502 (2006).

${ }^{20}$ R. Iacono, A. Bondeson, F. Troyon, and R. Gruber, Phys. Fluids B 2, 1794 (1990).

${ }^{21}$ H. P. Zehrfeld and B. J. Green, Nucl. Fusion 12, 569 (1972).

${ }^{22}$ K. C. Shaing, R. D. Hazeltine, and H. Sanuki, Phys. Fluids B 4, 404 (1992).

${ }^{23}$ L. C. Steinhauer, Phys. Plasmas 6, 2734 (1999).

${ }^{24}$ I. N. Sneddon, Elements of Partial Differential Equations (McGraw-Hill, New York, 1985).
${ }^{25}$ G. N. Throumoulopoulos, Phys. Plasmas 13, 122501 (2006).

${ }^{26}$ R. J. Akers, J. W. Ahn, G. Y. Antar, L. C. Appel, D. Applegate, C. Brickley, C. Bunting, P. G. Carolan, C. D. Challis, N. J. Conway, G. F. Counsell, R. O. Dendy, B. Dudson, A. R. Field, A. Kirk, B. Lloyd, H. F. Meyer, A. W. Morris, A. Patel, C. M. Roach, V. Rohzansky, A. Sykes, D. Taylor, M. R. Tournianski, M. Valovič, H. R. Wilson, K. B. Axon, R. J. Buttery, D. Ciric, G. Cunningham, J. Dowling, M. R. Dunstan, S. J. Gee, M. P. Gryaznevich, P. Helander, D. L. Keeling, P. J. Knight, F. Lott, M. J. Loughlin, S. J. Manhood, R. Martin, G. J. McArdle, M. N. Price, K. Stammers, J. Storrs, M. J. Walsh, MAST Team, and NBI Team, Plasma Phys. Controlled Fusion 45, A175 (2003).

${ }^{27}$ K. D. Marr, B. Lipschultz, P. J. Catto, R. M. McDermott, M. L. Reinke, and A. N. Simakov, Plasma Phys. Controlled Fusion 52, 055010 (2010).

${ }^{28}$ T. Tala, Y. Andrew, K. Crombé, P. C. de Vries, X. Garbet, N. Hawkes, H. Nordman, K. Rantamäki, P. Strand, A. Thyagaraja, J. Weiland, E. Asp, Y. Baranov, C. Challis, G. Corrigan, A. Eriksson, C. Giroud, M.-D. Hua, I. Jenkins, H. C. M. Knoops, X. Litaudon, P. Mantica, V. Naulin, V. Parail, K.-D. Zastrow, and JET-EFDA Contributors, Nucl. Fusion 47, 1012 (2007).

${ }^{29}$ A. R. Field, J. McCone, N. J. Conway, M. Dunstan, S. Newton, and M. Wisse, Plasma Phys. Controlled Fusion 51, 105002 (2009).

${ }^{30}$ P. Hirshman and D. J. Sigmar, Nucl. Fusion 21, 1079 (1981).

${ }^{31}$ W. M. Solomon, K. H. Burrell, R. Andre, L. R. Baylor, R. Budny, P. Gohil, R. J. Groebner, C. T. Holcomb, W. A. Houlberg, and M. R. Wade, Phys. Plasmas 13, 056116 (2006).

${ }^{32}$ A. Thyagaraja, P. J. Knight, M. R. de Baar, G. M. D. Hogeweij, and E. Min, Phys. Plasmas 12, 090907 (2005). 